\title{
Give and Take: Salmonella Enterica Alters Macrosteles Quadrilineatus Feeding Behaviors Resulting in Altered S. Enterica Populations and Distribution on Leaves
}

\author{
Victoria L. Harrod \\ University of Wisconsin - Madison \\ Russell Groves \\ University of Wisconsin - Madison \\ Ellie Guillemette \\ University of Wisconsin - Madison \\ Jeri Barak ( $\sim$ jeri.barak@wisc.edu ) \\ University of Wisconsin - Madison
}

\section{Research Article}

Keywords: Salmonella enterica, Hemipteran, Macrosteles quadrilineatus, feeding behaviors, S. enterica, populations, leaves

Posted Date: August 31st, 2021

DOI: https://doi.org/10.21203/rs.3.rs-828118/v1

License: (c) (i) This work is licensed under a Creative Commons Attribution 4.0 International License.

Read Full License 


\section{Abstract}

Hemipteran insects are ubiquitous inhabitants of the phyllosphere. Changes in microbial phyllosphere communities have recently been demonstrated following infestation by Macrosteles quadrilineatus (Aster Leafhopper). Although epiphytic Salmonella enterica populations naturally decline in the phyllosphere of plants, M. quadrilineatus infestation facilitated the growth of the bacterial pathogen populations. Here, we demonstrate that cellular damage by insect stylet penetration results in a localized beneficial niche on the leaf surface, leading to enhanced $S$. enterica populations. We measured $S$. enterica populations and colonization patterns on plants infested with Hemipterans with distinct feeding behaviors. $M$.

quadrilineatus infestation resulted in higher solute leakage and significantly greater bacterial populations than plants absent of insects. Following immigration via contaminated irrigation water, the highest populations of $S$. enterica are naturally found on the tips of tomato leaflets. We discovered $M$. quadrilineatus feeding preference altered the natural distribution of $S$. enterica populations, and that the presence of $S$. enterica altered the distribution of probing attempts. These findings elucidate how cellular damage resulting from insect feeding drives changes in bacterial colonization of the phyllosphere.

\section{Introduction}

Salmonella enterica, a human enteric bacterial pathogen, has recently been recognized as a member of the phyllosphere microbiome ${ }^{1,2}$. Unlike most members of this microbiome, the presence of $S$. enterica annually leads to food borne illness from the consumption of fresh, raw produce. In fact, cases of salmonellosis derived from the consumption of contaminated fresh produce has steadily increased over the last decade ${ }^{3}$. S. enterica colonization of the phyllosphere is reported to begin with contaminated irrigation water or direct application of raw (vs. composted) manure as a soil amendment ${ }^{4,5,6}$. Irrigation water is not only a conduit for $S$. enterica contamination directly to the phyllosphere, but can further spread the human pathogen from plant to plant via splash dispersal ${ }^{7}$. Here again, application of raw manure has been implicated as an avenue for contamination of agricultural fields but also has been reported to stimulate enteric bacterial growth on subsequent crops ${ }^{1}$. These human-mediated practices, among many others, aid $S$. enterica in achieving access to preharvest produce, thereby generating a scenario that may lead to foodborne illness.

While pathogen introduction to leaves preharvest is the first step in a sequence culminating in human illness, S. enterica populations have been observed to decline in the phyllosphere of healthy plants $8,9,10$. Hostile environmental conditions, such as direct UV radiation, desiccation, and a lack of nutrient availability, are a few of the limiting factors prompting bacterial populations to decrease over time. The high proportion of $S$. enterica outbreaks associated with consumption of fresh, raw produce, however, indicate that these bacteria have evolved to exploit several biological niches to successfully persist. Following contamination of the phyllosphere via irrigation water, $S$. enterica populations concentrate around glandular trichomes and stomates, two ubiquitous leaf structures that exude scarce metabolites or provide leaf internalization access respectively, each resulting in a beneficial niche for epiphytic 
bacteria ${ }^{11}$. Furthermore, $S$. enterica successfully persists near leaf regions altered by phytobacterial pathogens, such as Xanthomonas species ${ }^{12}$. These authors hypothesize that $S$. enterica uses nutrients made available during the plant infection process.

Just as a subset of phytobacterial pathogens were discovered as potential biological multipliers, we previously identified phytophagous insects as additional promoters for $S$. enterica survival in the phyllosphere ${ }^{13}$. Specifically, our lab uncovered that Macrosteles quadrilineatus (Aster leafhopper) infestation significantly promoted $S$. enterica populations and persistence overtime on both lettuce and tomato leaves ${ }^{9,13}$. Although this relationship was previously discovered, the mechanisms by which the insect facilitates the persistence of these bacterial populations has not yet been established.

We hypothesize that insect feeding alters the phyllosphere from a $S$. enterica inhospitable habitat to an inhabitable niche. Members of the Hemipteran order of insects utilize a narrow and segmented piercingsucking mouthpart, collectively composed of stylets, to feed on the phloem or xylem of plants. Although Hemipterans collectively share these mouthparts, different members of this important group employ unique probing and feeding strategies that elicit distinctive plant responses. For instance, the stylet of an aphid (Hemiptera, Stenorrhyncha, Aphidoidea) reaches the phloem via an intercellular pathway. After puncturing the epidermis, an aphid's stylet transiently probes and injects adjacent cells with watery saliva ${ }^{14,15}$ prompting upregulation of the salicylic acid pathway ${ }^{16,17}$. Contrastingly, leafhoppers (Hemiptera, Auchenorrhyncha, Cicadellidae) feed intracellularly by sieving through layers of cells to reach into the phloem, consequently upregulating the jasmonic acid pathway ${ }^{18}$. To date, the extent of cellular damage, elicited by feeding, has not been measured for Hemipterans. We hypothesize that these two modes of stylet penetration cause varying levels of cellular damage, and thus may uniquely alter the infested phyllosphere.

Here, we explore how differences between $M$. quadrilineatus and $M$. persicae feeding behaviors could influence the extent of cellular damage, and further how these differences may alter the phyllosphere for subsequent bacterial populations. We use $S$. enterica as a biological reporter of changes to the phyllosphere resulting from insect feeding, and lettuce and tomato plants were utilized as relevant plant systems for our experiments given their repeated association with domestic outbreaks of salmonellosis ${ }^{19,20}$. We hypothesize that preferred feeding locations of insects will experience higher levels of cellular damage, and in turn be associated with enhanced $S$. enterica populations ${ }^{21}$. To explore this hypothesis, we mapped preferential $S$. enterica colonization sites, preferred feeding locations of $M$. quadrilineatus, and examined whether earlier insect infestation influenced these distributions. In turn, we also investigated whether leaves previously contaminated with $S$. enterica would influence the feeding biology of $M$. quadrilineatus or M. persicae in the phyllosphere. Results from this study illustrate how unique hemipteran feeding behaviors can alter the phyllosphere and subsequent microbial community, with special reference to $S$. enterica.

\section{Materials And Methods}




\section{Bacterial Strains, Media, and Culture Conditions}

A kanamycin (Kan) resistant strain of $S$. enterica serovar Typhimurium 14028s was utilized and grown in a lysogeny broth (LB) at $37^{\circ} \mathrm{C}$, shaking at $200 \mathrm{rpm}$. Kan amended plates at concentrations of $50 \mathrm{mg} /$ liter were utilized to assess $S$. enterica populations. Bacteria from $-80^{\circ} \mathrm{C}$ freezer stocks were inoculated in LB broth and incubated, shaking overnight at $37^{\circ} \mathrm{C}$. S. enterica cultures were normalized to an optical density at $600 \mathrm{~nm}$ of 0.2 in sterile water. Inoculum preparations were verified by enumerating populations following serial dilution, plating on LB-Kan, and incubated overnight at $37^{\circ} \mathrm{C}$.

\section{Insect Rearing}

Colonies of Macrosteles quadrilineatus were maintained on oat seedlings (Avena sativa) under a constant temperature of $27^{\circ} \mathrm{C}$ and a 16:8 (L:D) photoperiod. A colony of Myzus persicae was provided by Jason Timothy Ingram and Dr. Stewart Gray (Cornell University) and maintained on turnip plants (Brassica rapa) under similar controlled conditions. Voucher specimens of adult female and male $M$. quadrilineatus and apterous M. persicae from our colony were deposited in the Wisconsin Insect Research Collection, University of Wisconsin (http://labs.russell.wisc.edu/wirc/).

\section{Plant assays}

Solanum lycopersicum (tomato, cv. Money Maker), and Lactuca sativa (lettuce, cv. Butterhead) seedlings were cultivated using Professional Growing Mix (Sunshine Redi-earth) in 6" pots held in a growth room maintained at a $16: 8(\mathrm{~L}: \mathrm{D})$ photoperiod and $24^{\circ} \mathrm{C}$ light and $19^{\circ} \mathrm{C}$ dark conditions. No plant material was collected. Seeds were bought commercially (available to the general public). Tomato plants were established and maintained for five weeks prior to all experiments, whereas lettuce plants were grown and utilized after six weeks. Six sets of $4.5 \mathrm{~cm}$ diameter clip cages, fashioned with insect-proof mesh, were fastened onto the abaxial (under) surface of two opposing leaves, three individually containing one adult $M$. quadrilineatus and the remaining three left empty as a control. Each clip cage was attached to the center of leaflets on each plant. Plants were held at a constant $24^{\circ} \mathrm{C}$ temperature with a $16: 8(\mathrm{~L}: \mathrm{D})$ photoperiod and were randomly assigned treatment groups indicating the length of infestation. At each infestation period of 24,48 , or 72 hours, individual M. quadrilineatus were removed, and leaf discs were excised from under each clip cage to assess for electrolyte leakage. Single experiments were carried out with apterous $M$. persicae (single insect per cage) on tomato plants. An additional experiment evaluated whether electrical conductivity measurements differ on tomato leaflets infested with singular or multiple aphids (3 individuals). Before infesting plants, individual $M$. quadrilineatus were collected with a respirator whereas a wet brush was used to transfer $M$. persicae. After the initial collection, insects were placed into a container over ice to impede movement, thereby facilitating the transfer into a clip cage. Insects were visually monitored for any movement immediately after plant application to ensure they were not injured during placement.

\section{Cellular Damage}


To analyze the extent of cellular damage associated with insect probing and feeding, estimates of electrolyte leakage were obtained by measuring electrical conductivity as previously described ${ }^{22}$. Briefly, a set of three comparable $10 \mathrm{~mm}$-diameter leaf discs from under clip cages with or without insects were placed in a single well of a 12-well tissue culture plate containing $4 \mathrm{ml}$ of sterile water. Plates were positioned on a rotating table at $50 \mathrm{rpm}$ for approximately $30 \mathrm{~min}$, acting as a wash step. This wash step prevented any leaf contaminants, such as remnant soil, from affecting conductivity measurements. Water from each well was subsequently removed and replaced with fresh, sterile water, and electrical conductance was immediately measured. Electrical conductance was measured by pipetting $1 \mathrm{ml}$ of the aqueous solution from sample wells onto an ECTestr11 + MultiRange electrical conductance probe to assess the extent of conductive electrolyte leakage, here used as a proxy for cellular damage. After the initial assessment of electrical conductance, sample plates were left on a lit bench at ambient temperature $\left(24^{\circ} \mathrm{C}\right)$ for 6 hours, after which a second and final conductivity measurement was taken. Differences in measured conductance between the two estimates were used for data analysis comparing each treatment group and used as a proxy for electrolyte leakage.

\section{Distribution of Salmonella enterica on the leaf phyllosphere}

To characterize the distribution of $S$. enterica on tomato and lettuce plants, attached leaflets and whole leaves, respectively were dip inoculated in a suspension of $S$. enterica. Replicate sets of tomato and lettuce plants were dip-inoculated for one minute in $450 \mathrm{ml}$ of sterile water or a $10^{8} \mathrm{CFU} / \mathrm{ml}$ suspension of $S$. enterica prepared as described above with the addition of $75 \mu \mathrm{L}$ of Sil-Wet. In each replicate, tip, middle and basal regions of whole leaves (lettuce) and leaflets (tomato) were randomized in a 2X2 factorial design, to receive either $S$. enterica suspensions or water controls. One-hour post-dip inoculation, clip cages were placed onto tip, middle and basal sections of leaflets or leaves for later assessments of electrolyte leakage assessment and $S$. enterica population enumeration. Water and $S$. enterica dipinoculated plants were then placed in clear, plastic bins held at $24^{\circ} \mathrm{C}$ temperature under a $16: 8$ (L:D) photoperiod and sampled seventy-two hours post-inoculation. In a complementary experiment designed to evaluate the influence of leaf angle on $S$. enterica distribution, water or $S$. enterica dip-inoculated plants were placed into a modified container with plastic ramps positioning tomato leaves at a $65^{\circ}$ vertical angle propping the tips of leaflets upwards and above the basal portions of leaves (e.g. petiole attachment). Prior to leaf excision for the two aforementioned experiments, each location (on the tip, middle and basal portions) was assigned a number and was entered into a random group generator, to prescribe the areas which would be used to measure $S$. enterica populations, and associated electrolyte leakage (https://www.randomizer.org).

To assess $S$. enterica populations, plants were sampled seventy-two hours after dip-inoculation. Specifically, one $10 \mathrm{~mm}$ diameter leaf disc was excised from under clip cages on either lettuce or tomato. Samples were individually homogenized in $500 \mu \mathrm{l}$ of sterile water using a cordless Dremel tool, and further diluted 1:10 in sterile water. Homogenates were immediately plated on LB-Kan, incubated overnight at $37^{\circ} \mathrm{C}$, and populations were enumerated after 24 hours. Electrolyte leakage was assessed 
three days after dip-inoculation as previously described. A total of 3 experimental replicates were completed.

\section{S. enterica, Plant, and Insect Interaction}

An additional experiment was performed to determine if the presence of M. quadrilineatus or M. precise altered the natural distribution of $S$. enterica populations or the magnitude of electrolyte leakage on tomato leaves. Groups of tomato plants were randomly assigned to treatment groups (water, or $S$. enterica) and arranged as a randomized complete block. One-hour post dip-inoculation, one clear hinged lid container ( $8 \times 53 / 4 \times 3$; Dart Container Corporation) was fastened onto a middle-aged leaflet. Clamshell containers were concurrently infested by five, adult $M$. quadrilineatus, or five apterous $M$. persicae, which were allowed to move freely around the entire leaflet, whereas a replicate set of uninfested clamshells remained empty. Replicate sets of $10 \mathrm{~mm}$ diameter leaf discs were sampled at 72 hours post-infestation and were randomly selected for assessments of $S$. enterica populations or electrolyte leakage (e.g. cellular damage). A total of 3 experimental replicates were completed.

To determine whether $S$. enterica could influence the feeding behavior of $M$. quadrilineatus, the distribution of salivary sheathes was observed on contaminated tomato leaves. Groups of four tomato plants were randomly assigned to the following inoculation groups: whole leaf water inoculation, whole leaf $S$. enterica inoculation, or $S$. enterica inoculated onto basal, middle or tip portions of select leaflets, and organized as a randomized complete block design with 3 experimental replicates. Regions uncontaminated by $S$. enterica were inoculated with sterile water. One hour post dip-inoculation, sets of 5 adult $M$. quadrilineatus were released into experimental clamshells and allowed access to whole leaves with different inoculation treatments. Following 72 hours of infestation, all insects were removed and whole leaflets were extracted, stained and cleared to enumerate salivary sheathes.

\section{Feeding and resting preference of $M$. quadrilineatus and $M$. persicae feeding on contaminated plants}

To determine the response of adult M. quadrilineatus'to leaf surfaces contaminated with S. enterica, two observational experiments were performed. In a first set of experiments, one middle-aged leaflet was entirely inoculated with sterile water, $S$. enterica, or both treatments on separate ends of leaves. One-hour post-inoculation, a modified clam shell container was affixed to encase each experimental treatment. Each cage was placed on a container at a height that would mimic the natural position of the leaflet and adjusted to ensure that the leaf did not touch the sides of the cage while still attached to the plant. Sets of five adult $M$. quadrilineatus per cage were released and allowed to move freely inside. Approximately 15 minutes post-infestation, a visual observation was made to assess the location (container, S. entericaor water-inoculated regions) of individual leafhoppers while also noting the position of insects on either abaxial or adaxial leaf surfaces. A total of 8 different visual assessments over 2 hours were conducted 
for each set of leafhoppers, leading to 16 observations per treatment group for one experimental replicate. A total of 5 experimental replicates were completed.

To further define whether $S$. enterica influenced $M$. quadrilineatus and $M$. persicae resting preferences, observations of adult insects were made in terms of their positions across leaflets or the experimental cage. Observations (basal, middle or tip) for sets of M. quadrilineatus (5 per plant) were recorded at 2-, 24-, and 48 hours post infestation on tomato leaflets inoculated exclusively at the base, middle or tip, or entirely inoculated with $S$. enterica or water. Observations ( $S$. enterica or water) for sets of M. persicae (1 per clip cage) were recorded at 24-, 48- and 72 hours post infestation on $S$. enterica or water inoculated halves of leaflets. The location of $S$. enterica inoculations were randomly assigned to leaf areas.

Differences in observed times and the alternative inoculation style between insect species was chosen to accommodate the smaller and lesser mobile apterous life stage of $M$. persicae, compared to the larger bodied and more mobile $M$. quadrilineatus. Groups of 4 plants were utilized for each treatment group with 3 experimental replicates. At the conclusion of these experiments, leaflets from M. quadrilineatus infested plants were removed and stained in an effort to count salivary sheathes 72 hours following insect exposure.

\section{Salivary Sheath Staining and Clearing Procedure}

To enumerate salivary sheaths associated with adult M. quadrilineatus feeding, experimental leaflets were extracted, and subsequently stained at room temperature for 20 to 24 hours with $0.2 \%$ acid fuchsin in a $1: 1(\mathrm{vol} / \mathrm{vol})$ solution of $85 \%$ glacial acetic acid and $95 \%$ ethanol, otherwise known as McBryde's acid fuchsin stain ${ }^{23,24}$. To remove chlorophyll and clear tissues, leaflets were soaked in $95 \%$ ethanol for 30 minutes, replacing the stained liquid with new ethanol every 10 minutes. Leaflets were then heated in a $1: 1: 1$ ( $\mathrm{vol} / \mathrm{vol} / \mathrm{vol})$ solution in $95 \%$ ethanol, $85 \%$ glacial acetic acid, and water, and individually boiled for 8 to 10 minutes to appear translucent. Salivary sheathes of individual leaflets were visually quantified under an Olympus SZ60 Stereoscope.

\section{Statistical Analysis}

Student's t-tests were performed to compare estimates of electrical conductivity between the two experimental taxa ( $M$. quadrilineatus and $M$. persicae), together with bacterial contaminated and water inoculated treatments at 24,48 , or 72 hours. A one-way, analysis of variance (ANOVA) was used to assess if $S$. enterica populations or electrical conductance measurements varied among regions on leaves/leaflets including basal, middle, or tip regions. Additionally, ANOVA was used to determine the distribution of salivary sheathes across uniquely inoculated tomato leaflets. Interpolation was used to visualize estimated $S$. enterica populations outside of the pre-determined leaf excision points using the 'lattice' and 'akima' packages on R-Studio. An analysis of covariance (ANCOVA) was used to analyze the proportion of resting $M$. quadrilineatus and $M$. persicae on tomato or container locations when $S$. enterica was both present and absent in select treatments. M. quadrilineatus preference for uniquely S. enterica inoculated surfaces, or alternative surfaces (water, or cage), was determined using a likelihood ratio chisquare test. 


\section{Results}

M. quadrilineatus infestation (intracellular penetration) results in greater cellular damage than uninfested plants.

To further investigate how different feeding styles alter the phyllosphere, we analyzed the extent of electrolyte leakage (using electrical conductivity as a proxy) on tomato plants in response to intracellular or intercellular penetration employed by leafhoppers and aphids, respectively. Tomato plants infested with leafhoppers had significantly higher levels of measured electrical conductivity at 24 hours postinfestation (hpi) when compared to plants with no insects ( $P<0.0005$; Fig. 1a). After 24 hpi, measurements of electrical conductivity were not significantly different on tomato plants infested by $M$. quadrilineatus, or without insects ( $P>0.05$; Fig. 1a). In the presence of aphids, measurements of electrical conductivity were not significantly different between plants with or without insects at the measurement timepoints of 24,48 , or $72 \mathrm{hpi}(P>0.05$; Supplemental Fig. S1). A complimentary experiment determined that increasing aphid populations, from one individual to three, did not influence the extent of electrolyte leakage on tomato plants ( $P=0.398$; Supplemental Fig. S2). To determine if this pattern of electrolyte leakage following leafhopper infestation was independent of host, we tested lettuce plants and continued to observe higher electrical conductivity estimates at 24,48 , and $72 \mathrm{hpi}(P<0.0001$; Fig. 1b). Electrical conductivity values were not measured in response to aphids on lettuce.

S. enterica populations naturally accumulate at the tips of tomato leaflets.

To better understand how leafhopper feeding alters the distribution of S. enterica in the phyllosphere, we examined how bacterial populations changed across the leaf surface. First, the natural distribution of $S$. enterica on lettuce and tomato plants was determined (Fig. 2). Tomato leaflets supported significantly higher $S$. enterica populations at the tip of leaflets, compared to samples measured from the basal regions $(P<0.0007$; Fig. 2a). Measurements of electrical conductivity at the base of tomato leaflets were higher, but not significantly different than samples collected in the middle or tip regions of leaflets $(P=$ 0.0626; Supplemental Fig. S3a). To test whether $S$. enterica population distribution across the tomato leaflet is influenced by gravity, a complementary experiment was designed to disrupt the natural tendency of leaflets to droop and consequentially result in liquid collecting on leaflet tips. When leaflets were placed in a more upright position ( $65^{\circ}$ upward angle) after the dip-inoculation, the highest accumulation of bacterial populations shifted to the base ( $P=0.0068$; Supplemental Fig. S4), whereas measured electrical conductance remained higher, but not significantly different, at the basal region of the leaf ( $P=$ 0.061; Supplemental Fig. S5). On lettuce, $S$. enterica populations remained somewhat uniform across the leaf $(P=0.87$; Fig. $2 b)$ while the base of lettuce plants exhibited significantly higher electrical conductivity than the middle or tip regions ( $P<0.001$; Supplemental Fig. S3b). With improved knowledge of where $S$. enterica preferentially colonizes the phyllosphere, we could examine if leafhopper infestation alters bacterial distribution. 
Insect infested tomato leaflets had the greatest electrical conductivity and enhanced S. enterica populations.

To test the hypothesis that cellular damage, prompted by leafhopper intracellular penetration, facilitates fundamental changes in the phyllosphere, we measured $S$. enterica populations in response to $M$. quadrilineatus infestation (Fig. 3). After 72 hpi, S. enterica populations were approximately half a log higher on tomato plants infested with leafhoppers than $S$. enterica inoculated plants without insects, consistently indicating that leafhopper infestation enhances $S$. enterica populations $(P<0.0001$; Fig. 3a). Similarly, plants infested by leafhoppers and contaminated with $S$. enterica exhibited significantly greater estimates of electrolyte leakage than plants without insects ( $P=0.0020$; Fig. 3b), and had higher, but not significantly different, electrical conductivity measurements than infested plants treated with water only. Infested plants had a roughly uniform distribution across the middle and tip regions of leaflets indicating a shift in expected natural bacterial populations. Although the $S$. enterica population was similar at the tip for either infested or leaves without insects, the base and middle locations of infested leaflets had significantly higher $S$. enterica than the same locations on uninfested leaflets $(P<0.005$; Fig. $4 a-b)$, suggesting that insect activity increased the local $S$. enterica populations in these regions of the leaflet. Electrical conductivity was not significantly different across a leaflet within any treatment group and was similar between the tip, middle, and basal regions of infested $S$. enterica tomato plants $(P>0.05$; Supplemental Fig. S6). Greater cellular damage on infested, $S$. enterica inoculated leaflets prompted an additional set of experiments to determine whether $M$. quadrilineatus feeding and resting preference was influenced by $S$. enterica or water-inoculated leaves. Measurements of $S$. enterica populations and electrical conductivity within a randomized block design were similarly measured for $M$. persicae, yet no significant differences between infested and uninfested plants were observed $(P>0.05$; Supplemental Fig. S7).

Adult M. quadrilineatus prefer water inoculated surfaces, over those inoculated with S. enterica.

When provided a choice, adult $M$. quadrilineatus discriminated between non-plant and plant surfaces over a 2-hour period, landing more frequently on water inoculated areas than on $S$. enterica inoculated areas $(P<0.005 ;$ Supplemental Fig. S8). When exposed to partially or entirely inoculated leaflets for a greater duration, $M$. quadrilineatus were observed to explore leaf surfaces at 2-hours post infestation but migrated away from leaflets and onto the experimental cage after 48-hours of exposure to $S$. enterica $(P<$ 0.001; Fig. 5). Similar to M. quadrilineatus, sets of $M$. persicae were also exposed to inoculated tomato leaflets over a $72 \mathrm{hr}$ experimental interval. While a pattern of emigration from inoculated leaflets and towards the cage of the experimental arena emerged over the 72-hour period of infestation, apterous $M$. persicae indicated no significant substrate preference ( $P>0.05$; Supplemental Fig. S9).

Salivary sheath distribution is dependent upon S. enterica presence.

To further define M. quadrilineatus'preferred feeding sites in relation to the presence of $S$. enterica, the presence of salivary sheathes across water and $S$. enterica inoculated leaflets were observed. Across all inoculation treatment groups, salivary sheathes were observed to be significantly less abundant on 
primary and secondary veins indicating a predominant preference for tertiary, or lesser, veins $(P<0.0001$; Supplemental Fig. S10). As a result, salivary sheathes located on primary and secondary veins were excluded from statistical comparisons of salivary sheath distribution across the basal, middle and tip regions of uniquely inoculated leaflets. Salivary sheathes were most regularly found on the middle of leaflets inoculated at the base or tip, and on leaflets inoculated entirely with $S$. enterica or water $(P<$ 0.0001 ; Fig. 6). However, inoculation of $S$. enterica exclusively on the middle portions of leaflets, their preferred feeding location, resulted in a shift of salivary sheath distribution as significantly more salivary sheathes were found at the base of leaflets than the middle ( $P=0.0237$; Fig. 6$)$. This finding demonstrates that even limited presence of $S$. enterica on a leaflet alters the preferred probing/feeding locations of adult $M$. quadrilineatus.

\section{Discussion}

The now frequent, reoccurrence of foodborne illness cases associated with consumption of fresh produce requires an in-depth assessment of environmental factors that increase the risks of continued outbreaks. In our current study, we examined the population dynamics of a foodborne pathogen through an entomological perspective, analyzing the tri-trophic interactions between $S$. enterica, plants, and phytophagous insects. Specifically, we investigated how changes in the phyllosphere resulting from unique insect feeding styles, impacted the longevity and persistence of $S$. enterica populations on the leaf surface.

Previous literature demonstrated that insects can manipulate human enteric bacterial pathogen populations directly, and indirectly. Within poultry dominated environments, cockroaches may mechanically transmit $S$. enterica by traversing from contaminated egg surfaces to uncompromised substrates, consequently facilitating the movement of bacteria ${ }^{25}$. Seaweed flies, intimately associated with decaying and pathogenic seaweed beds, excrete viable bacterial populations within intertidal zones, enhancing the potential transmission of $E$. coli ${ }^{26}$. Within commercial agriculturally centered models, $M$. quadrilineatus enhanced transmission of $S$. enterica from contaminated leaves to clean leaves or adjacent plants ${ }^{13}$. Furthermore, excretion of viable $S$. enterica from $M$. quadrilineatus has also been documented ${ }^{27}$. Yet, how phytophagous insects influence this increase of $S$. enterica persistence on leaves remains mostly unexamined.

In earlier studies, we observed that only $M$. quadrilineatus infestation led to an increase in S. enterica persistence, but no observed benefit occurred following $M$. persicae infestation ${ }^{8}$. The findings of this investigation point towards differences between the inter- and intracellular penetrative styles of feeding between these two taxa, and the resulting effects these styles may hold for $S$. enterica population dynamics within the phyllosphere. While both insects possess similar mouthpart structures, collectively referred to as stylets, their modes of reaching vascular tissues are very distinct. Aphids, or intercellular feeders, begin probing at the junction of two epidermal cells and guide their stylet through intercellular spaces in the mesophyll and towards vascular bundles ${ }^{28}$. Leafhoppers, considered as intracellular 
feeders, similarly begin feeding at a cell junction, but distinctly pierce through leaf mesophyll to reach the phloem ${ }^{29}$. Comparisons of the electrical conductivity response of leaflets infested by inter- and intracellular penetration revealed that $M$. quadrilineatus infestation elicits a greater magnitude of electrolyte leakage, and consequently greater cellular damage than M. persicae on tomato plants (Fig. 1; Supplemental Fig. S1). Furthermore, our current study demonstrated that plants contaminated by $S$. enterica and infested with $M$. quadrilineatus had the highest overall populations of bacteria and resulted in the greatest magnitude of electrolyte leakage (measured as electrical conductance) (Fig. 3a-b). In addition to their distinct feeding behaviors, leafhoppers possess a stylet bundle 5-times wider than those found on aphids ${ }^{30,31}$. To compensate for the lesser stylet, we investigated the influence of higher aphid populations in a complementary experiment, yet found no measurable impact on enhanced electrolyte leakage, or cellular damage (Supplemental Fig. S2). Taken together, the wider stylet paired with intracellular lacerating types of feeding behavior by M. quadrilineatus may partially explain the enhanced magnitude of cellular damage on the phyllosphere of tomato plants (Fig. 1). These findings lead us to conclude that cellular damage induced by $M$. persicae probing behaviors does not manipulate the phyllosphere to the same extent as M. quadrilineatus.

As previously mentioned, $S$. enterica and $M$. quadrilineatus co-habitation on the same leaflet resulted in higher $S$. enterica populations and measured electrolyte leakage (aka cellular damage) compared to water inoculated leaflets with or without insects (Fig. 3a-b). These elevated levels of cellular damage are likely the result of greater probing frequencies and may indicate an unfavorable feeding environment for the insect, prompting them to more frequently probe and search for alternative food sources. Previous studies identified clusters of gustatory neurons, which when combined, functionally create taste receptors within insects ${ }^{32}$. When encountering food contaminated by lipopolysaccharides (LPS), a ubiquitous component found on gram-negative bacterial cells, Drosophila melanogasternot only avoids E. colicontaminated foods but also commence a hygienic grooming regimen ${ }^{33}$. This prompted behavior suggests that some insects can discriminate between LPS contaminated and non-contaminated food sources via gustatory cues. Although many of these studies focus on insects with sponging-sucking mouthparts, such as flies, a genome analysis identified both odorant and gustatory receptor genes in aphid and mosquito genomes, both of which possess piercing-sucking mouthparts comparable to that of $M$. quadrilineatus ${ }^{34}$. In our experiments where we confined $M$. quadrilineatus and $S$. enterica together in more proximal environments, we propose that the adult leafhoppers could encounter higher traces of LPS and may modify their normal feeding behavior as a consequence. Due to the restricted movement in these instances, we surmise the heightened magnitude of electrolyte leakage is driven by a constant search for a non-contaminated substrate and thus, heightened occurrences of probing for a new food source on S. enterica inoculated plant (Fig. 3b). To further evaluate whether $S$. enterica presence alters $M$. quadrilineatus' movement, we provided $M$. quadrilineatus with contaminated (S. enterica) and noncontaminated (sterile water) tomato leaflet surfaces and monitored their resting or feeding locations every 15 minutes thereafter for over a two-hour period. Throughout the time course of these observational experiments, a pattern of substrate discrimination occurred (Supplemental Fig. S8). Most insects initially landed on the plastic container housing the experiment, but over time began to immigrate more often to 
water-inoculated surfaces than those with $S$. enterica. In a complementary experiment, adult M. quadrilineatus exposed to tomato leaflets inoculated at either tip or basal regions of leaves similarly preferred water inoculated regions at 2 hours post exposure, but predominantly emigrated to the experimental container walls after 48 hours (Fig. 5). Altogether, leaflets entirely or partially inoculated with S. enterica were less frequently visited at the last time point (72 hours post infestation), whereas leaflets inoculated solely with water were occupied throughout the experiment. Contrasting this behavior, apterous $M$. persicae exhibited no preference between $S$. enterica or alternative surfaces (Supplemental Fig. S9). This lack of substrate preference may result from the largely sessile lifestyle of aphids, in contrast to more mobile and alate leafhoppers. These avoidance behaviors by M. quadrilineatus in response to $S$. enterica inoculated leaflets suggest a capability of recognizing contaminated substrates similar to the responses described for $D$. melanogaster.

To evaluate the extent by which $M$. quadrilineatus might influence the distribution of bacterial populations across leaflets, we first defined the distribution of $S$. enterica and the magnitude of electrolyte leakage across tomato and lettuce leaves in the absence of any insects. Morphological features between pre-reproductive lettuce and tomato plants are vastly distinct and were hypothesized to impact the distribution of bacterial populations and electrolyte leakage. In our study, the leaf tips were the lowest positioned part of tomato leaflets and exhibited half a log higher $S$. enterica populations in comparison to basal regions (Fig. 2b). Here again, the nominal architecture of tomato leaves results in a natural 'drooping' of fully expanded leaf tips. In a complementary experiment, tomato leaflets were modified to reverse this normal positioning of leaf tips to basal regions, and we did observe a corresponding redistribution of $S$. enterica where accumulations were enhanced on basal portions of leaves (Supplemental Fig S4). These findings suggest that during the application of an aqueous solution - such as contaminated irrigation water or even foliar-applied crop inputs - factors including gravitational force may influence aggregations of aqueous solutions on leaves ${ }^{35}$. This suite of findings identified leaf positioning and morphology, in conjunction with gravitational forces, as dominant influences of $S$. enterica population distribution across tomato leaflets while unaffecting the degree of electrical conductivity estimates, or associated electrolyte leakage of leaf electrolytes (Supplemental Fig. S3b). Despite $S$. enterica populations being highest at the tips of unaffected leaflets, bacterial populations were comparable at the tip and middle portions of leaflets only after adult M. quadrilineatus infestation, suggesting an insect mediated influence (Fig. 4). To this finding, we hypothesized that leafhopper feeding is not uniform or homogeneous across whole leaflets and that the distribution of leaf vascular bundles may influence where adult leafhoppers find preferential feeding sites. The diameter of primary and secondary angiosperm vascular bundles typically narrows from the base to the tip of leaves, presumably to maximize the efficiency of hydraulic conductivity using adhesive and cohesive forces ${ }^{36,37}$. This natural tapering of vascular structures at the tips of leaves provides piercing-sucking insects with some limitations in the number of ideal feeding locations and we hypothesize that the variation in the dendritic nature of leaf venation may alter the distribution of $M$. quadrilineatus feeding sites, explaining the higher $S$. enterica populations in the middle of infested tomato leaflets ${ }^{38}$. In addition to frequently observing leafhoppers in middle portions of leaflet regions, salivary sheathes were also predominantly found in 
similar regions of water-inoculated leaflets indicating preferences for these vascular bundles across leaflets (Fig. 6). Despite being their preferred feeding site, S. enterica inoculation at the middle of leaflets appeared to influence adult $M$. quadrilineatus towards feeding at the non-contaminated basal and tip regions, away from the $S$. enterica middle regions (Fig. 5). Similarly, leaflets partially inoculated at the base and tip had the least amount of salivary sheathes at their base and tip, respectively. This consistent pattern of probing avoidance of contaminated regions suggests that $M$. quadrilineatus may exhibit discriminatory behaviors against leaflets where $S$. enterica was present, indicating that even limited exposure to $S$. enterica holds potential to alter natural feeding behaviors as seen on water inoculated leaflets.

Although $M$. quadrilineatus exhibited avoidance behaviors of partially inoculated leaflets, their mobile lifestyle illustrates their potential as a biological multiplier for $S$. enterica. During their exposure to partially inoculated leaflets, salivary sheathes were identified at the base, middle and tip, although nonuniformly, suggesting an exploratory behavior (Fig. 6). This movement across S. enterica contaminated leaflets and the subsequent aversion suggest a likelihood for emigrating to alternative food sources (Fig. 7). Logically, if $M$. quadrilineatus have previously encountered $S$. enterica contaminated leaves or plants, then mechanical transmission of bacteria could further exacerbate the likelihood of $S$. enterica dissemination within contaminated agricultural crops and promote the possibility of food borne outbreaks.

Within this study, we aimed to characterize insect feeding behaviors which could directly enhance $S$. enterica populations on tomato leaflets. Although we directly focused on cellular damage by stylet penetration, a suite of other phenomena (i.e. honeydew production and plant immunity regulation) occurring in tandem necessitate further investigation. While these biological factors likely co-occurred, we identified prominent insect-mediated interactions involving cellular damage, unique insect feeding behaviors, and $S$. enterica populations, thereby demonstrating intracellular stylet penetration by $M$. quadrilineatus as a beneficial insect behavior for $S$. enterica persistence. Furthermore, we demonstrated that plant morphology directs the distribution of bacterial populations when dispersed aqueously yet may be manipulated in the presence of $M$. quadrilineatus due to increased stylet probing at preferred feeding sites. Although our results were collected under laboratory conditions, our findings elucidate how insects interact within the phyllosphere, and in turn, influence $S$. enterica population dynamics.

\section{Declarations}

\section{Acknowledgments}

We thank Benjamin Bradford for providing guidance on modeling figures and statistical analysis. Funding was provided by USDA-NIFA 2016-67017-24422 and the Food Research Institute at the University of Wisconsin-Madison.

\section{Author Contributions}


V.L, R.G, and J.B conceptualized the design of the study and provided revisions of the manuscript; V.L and E.G curated data and conducted formal analysis.

\section{Competing Interests}

The authors declare no competing interests.

\section{References}

1. Jechalke, S., Schierstaedt, J., Becker, M., Flemer, B., Grosch, R., Smalla, K., Schikora, A. 2019. Salmonella Establishment in Agricultural Soil and Colonization of Crop Plants Depend on Soil Type and Plant Species. Front Microbiol. 3389/fmicb.2019.00967

2. Zarkani, A. A., Schierstaedt, J., Becker, M., Krumwiede, J., Grimm, M., Grosch, R., Jechalke, S., Schikora, A. 2019. Salmonella adapts to plants and their environment during colonization of tomatoes. FEMS Microbiology Ecology. 1093/femsec/fiz152.

3. Callejon, R. M., Rodriguez-Narango, I., Ubeda, C., Hornedo-Ortega, R., Garcia-Parrilla, M. C., Troncoso, A. M. Reported Foodborne Outbreaks Due to Fresh Produce in the United States and European Union: Trends and Causes. Pathogens and Disease. 12: 32-38. 10.1089/fpd.2014.1821.

4. Hruby, C. E., Soupir, M. L., Moorman, T. B., Pederson, C.H., Kanwar, R.S. 2018. Salmonella and Fecal Indicator Bacteria Survival in Soils Amended with Poultry Manure. Water Air Soil Pollut. 229:32. 10.1007/s11270-017-3667-z.

5. Islam, M., Morgan, J., Doyle, M. P., Phatak, S. C., Millner, P., Jiang, X. 2004. Persistence of Salmonella enterica serovar Typhimurium on lettuce and parsley and in soils on which they were grown in fields treated with contaminated manure composts or irrigation water. Foodborn Pathog. Dis. 1:27-35.

6. Liu, H., Whitehouse, C. A., Li, B. 2018. Presence and Persistence of Salmonella in Water: The Impact on Microbial Quality of Water and Food Safety. Front Public Health 6:159.

10.3389/fpubh.2018.00159.

7. Cevallos-Cevallos, J. M., Danyluk, M. D., Gu, G., Vallad, G. E., van Bruggen, A. H. C. 2012. Dispersal of Salmonella Typhimurium by rain splash onto tomato plants. Journal of Food Protection. 75:472479.

8. Soto-Arias, J. P., Groves, R. L., and Barak, J. D. 2013. Interaction of phytophagous insects with Salmonella enterica on plants and enhanced persistence of the pathogen with Macrosteles quadrilineatus infestation or Frankliniella occidentalis feeding. PLoS One 8: e79404. 10.1371/journal.pone.0079404

9. Cowles, K. N., Groves, R. L., Barak, J. D. 2018. Leafhopper-induced activation of the jasmonic acid response benefits Salmonella enterica in a flagellum-dependant manner. Front Microbiol. 10.3389/fmicb.2018.01987

10. Chiu, Y., Shen, C., Farnham, M. W., Ku, K. 2020. Three-dimensional epicuticular wax on plant surface reduces attachment and survival rate of Salmonella during storage. Postharvest Biol Tec. 
10.1016/j.postharvbio.2020.111197.

11. Barak, J. D., Kramer, L. C., Hao, L. Y. 2011. Colonization of tomato plants by Salmonella enterica is cultivar dependent, and type 1 trichomes are preferred colonization sites. Appl Environ Microbiol. 77:498 - 504. 10.1128/AEM.01661-10.

12. Potnis, N., Soto-Arias, J. P., Cowles, K. N., van Bruggen, A. H., Jones, J. B., Barak, J. D. 2014. Xanthomonas perforans colonization influences Salmonella enterica in the tomato phyllosphere. Appl Environ Microbiol 80: 3173 - 3180. 10.1128/AEM.00345-14.

13. Soto-Arias, J. P., Groves, R., and Barak, J. D. 2014. Transmission and Retention of Salmonella enterica by Phytophagous Hemipteran Insects. Appl Environ Microbiol 80: 5447 - 5456.

10.1128/AEM.01444-14

14. Schwarzkopf, A., Rosenberger, D., Niebergall, M., Gershenzon, J., Kunert, G. 2013. To Feed or Not to Feed: Plant Factors Located in the Epidermis, Mesophyll, and Sieve Elements Influence Pea Aphid's Ability to Feed on Legume Species. PLOS One 8(9): e75298.

15. Miles, P. W. 1999. Aphid saliva. Biol Rev 74:41 - 85. doi: 10.1111/j.1469-185X.1999.tb00181.x

16. Mohase, L., van der Westhuizen, A. J. 2002. Salicylic acid is involved in resistance responses in the Russian wheat aphid-wheat interaction. Plant Physiol. 159:585-590.

17. Kuśnierczyk, A., Winge, P., Jørstad, T. M., Troczyńska, J., Rossiter, J. T., Bones, A. M. (2008) Towards global understanding of plant defence against aphids-timing and dynamics of early Arabidopsisdefence responses to cabbage aphid (Brevicoryne brassicae) attack. Plant Cell Environt. 31:1097-1115

18. Hunter, W. B., Backus, E. A. 1989. Mesophyll-feeding by the potato leafhopper, Empoasca fabae (Homoptera: Cicadellidae): results from electronic monitoring and thin-layer chromatography. Environ Entomol. 18:465-72

19. Centers for Disease Control and Prevention (CDC). 2007. Multistate outbreaks of Salmonella infections associated with raw tomatoes eaten in restaurants - United States, 2005 - 2006. MMWR Morb Mortal Wkly Rep. 56(35): 909-911.

20. Cummings, K., Barrett, E., Mohle-Boetani, J. C., Brooks, J. T., Farrar, J., Hunt, T., Fiore, A., Komatsu, K., Werner, S. B., Slutsker, L. 2001. A multistate outbreak of Salmonella enterica serotype Baildon associated with domestic raw tomatoes. Emerg Infect Dis. 7: 1046 - 1048. 10.3201/eid0706.010625.

21. Harrod, V. L., Groves, R. L., Maurice, M. A., Barak, J.D. (2021) Frankliniella occidentalisfacilitate Salmonella enterica survival in the phyllosphere. PLoS ONE 16(2): e0247325. 10.1371/journal.pone.0247325

22. Kessens, R., Sorensen, N., Kabbage, M. 2018. An inhibitor of apoptosis (SfIAP) interacts with SQUAMOSA promoter-binding protein (SBP) transcription factors that exhibit pro-cell death characteristics. Plant Direct. 10.1002/pld3.81.

23. McBryde. 1936. A Method of Demonstrating Rust Hyphae and Haustoria in Unsectioned Leaf Tissue. Am J Bot. 23: 686 - 688. 
24. Yang, Y., Beattie, G. A. C., Spooner-Hart, R. N., Huang, M., Barchia, I., Holford, P. 2012. Influences of leaf age and type, non-host volatiles, and mineral oil deposits on the incidence, distribution, and form of stylet tracks of Diaphorina citri. Entomol Exp Appl. 1111/eea.12046.

25. Kopanic, R. J., Sheldon, B. W., Wright CG. 1994. Cockroaches As Vectors of Salmonella: Laboratory and Field Trials. Journal of Food Protection. 10.4315/0362-028X-57.2.125.

26. Swinscoe, I., Oliver, D. M., Gilburn, A. S., Quilliam, R. S. 2018. The seaweed fly (Coelopidae) can facilitate environmental survival and transmission of E. coli 0157 at sandy beaches. Journal of Environmental Management. 223: 275 - 285. 10.1016/j.jenvman.2018.06.045.

27. Dundore-Arias, J.P., Groves, R. L., Barak, J. D. 2015. Influence of prgH on the persistence of ingested Salmonella enterica in the leafhopper Macrosteles quadrilineatus. Environ. Microbiol. 81:6345-6354.

28. Tjallingii, W. F. 2006. Salivary secretions by aphids interacting with proteins of phloem wound responses. J Exp Bot. 57: 739 - 745.

29. Pollard, D. G. 1969. Directional control of the stylets in phytophagous Hemiptera. Proc R Entomol Exp Appl. 44: 173 - 185.

30. Pollard, D. G. 1972. The stylet structure of a leafhopper (Eupteryx melissae Curtis: Homoptera, Cicadellidae). Journal of Natural History. 6: $261-271$.

31. Forbes, A. R. 1969. The stylets of the green peach aphid, Myzus persicae (Homoptera: Aphididae). Can Ent. 101: 31 - 41.

32. Soldano, A., Alpizar, A.Y., Boonen, B., Franco, L., Lopes-Requena, A., Liu, G., Mora, N., Yaksi, E., Voets, T., Vennekens, R., Hassan, B. A., Talavera, K. 2016. Gustatory-mediated avoidance of bacterial lipopolysaccharides via TRPA1 activation in Drosophila. eLIFE. 10.7554/eLife.1313

33. Yanagawa, A., Guigue, A. M. A., Marion-Poll, F. 2014. Hygienic grooming is induced by contact chemicals in Drosophila melanogaster. Front Behav Neurosci. 10.3389/fnbeh.2014.00254.

34. Isono, K., Morita, H. 2010. Molecular and cellular designs of insect taste receptor system. Cell. Neurosci. 10.3389/fncel.2010.00020

35. Yu, Y., Jung, H., Cho, H. 1999. A new water droplet model using metaball in the gravitational field. Comput Graph.1016/S0097-8493(99)00031-X.

36. McKown, A. D., Cochard, H., Sack, L. 2009. Decoding Leaf Hydraulics with a Spatially Explicit Model: Principles of Venation Architecture and Implications for Its Evolution. Am Nat. 1086/650721.

37. Sack, L., Scoffoni, C. 2013. Leaf venation: structure, function, development, evolution, ecology and applications in the past, present and future. New Physiol. 198: $983-1000$.

38. Peeters, P. J. 2002. Correlations between leaf structural traits and the densities of herbivorous insect guilds. Biol J Linn Soc. 77: $43-65$.

\section{Figures}



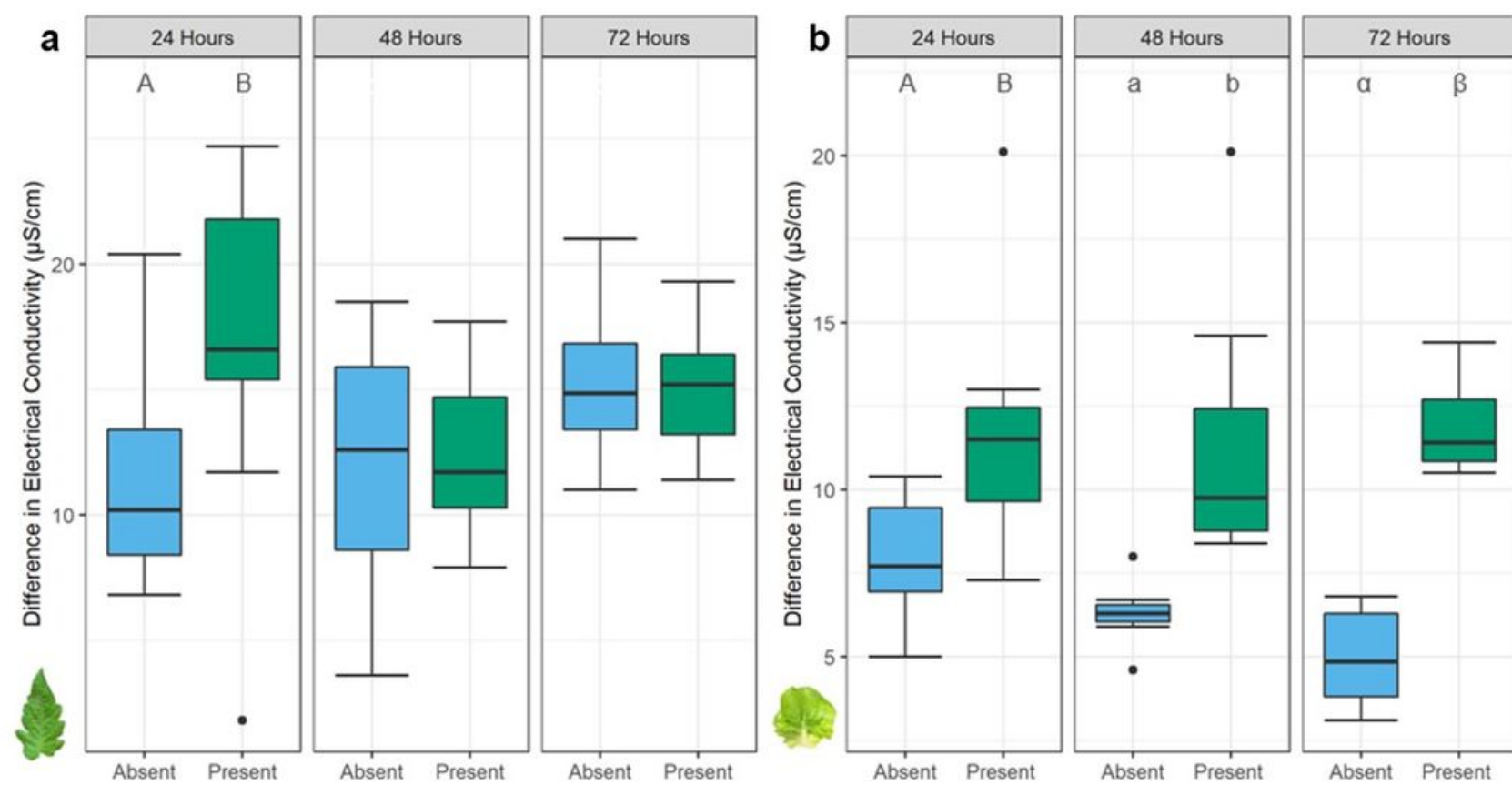

Status of Insect Infestation

追 Absent

官 Present

\section{Figure 1}

Infestation by Macrosteles quadrilineatus leads to an increase in measured cellular damage on tomato (a) and lettuce (b) leaves. When compared to uninfested areas, electrolyte leakage was significantly higher on tomato leaflets (a) infested for 24 hours, and on lettuce leaves (b) infested for 24,48 , and 72 hours $(P<0.05)$. Three clip cages were fastened onto a middle leaf each containing one leafhopper (green), and additional three clip cages remained empty (blue) on an opposing leaflet. Electrical conductance was calculated by subtracting the final from the initial measurement for damaged and undamaged leaf discs and were used to evaluate the extent of electrolyte leakage over six hours. Letters above boxplot indicate significant differences between treatment groups within a single time point $(\mathrm{P}<$ 0.05). A student's t-test was used to assess significance between samples from infested or non-infested clip cages. Singular dots represent an outlier point. 

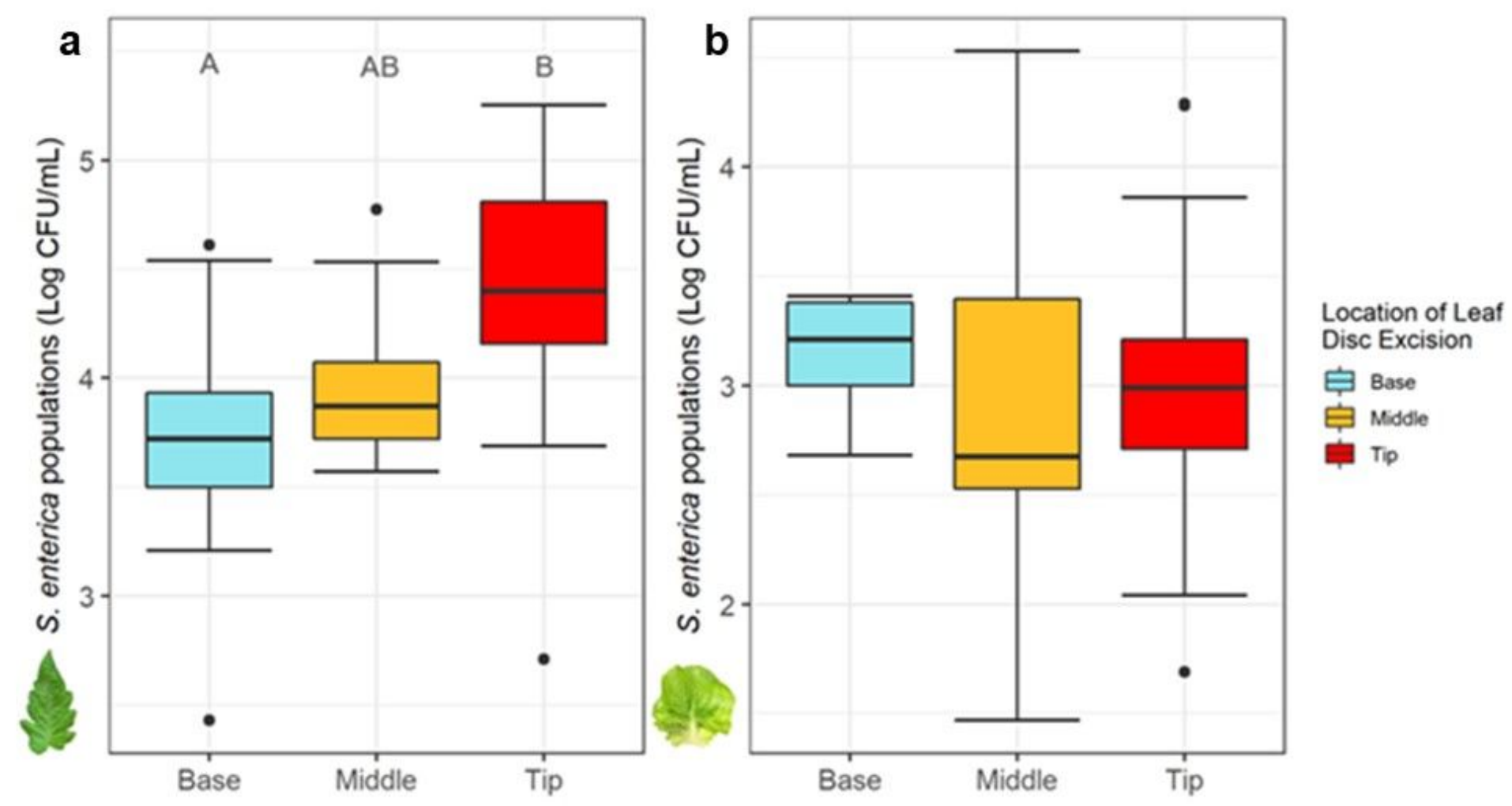

Figure 2

Tomato leaf architecture impacts the distribution of S. enterica populations, unlike lettuce. Salmonella enterica populations were significantly greater at the tip of tomato leaflets than the base (Fig. $2 a)(P<$ 0.05), whereas lettuce leaves have a uniform bacterial distribution (Fig. 2b). Leaf discs were excised from pre-determined locations from the basal (blue), middle (orange), and tip (red) regions of leaves. Letters above boxplots indicate significant differences between treatment groups within each experiment $(P<$ 0.05), as detected by a one-way ANOVA. Singular dots represent an outlier point. 

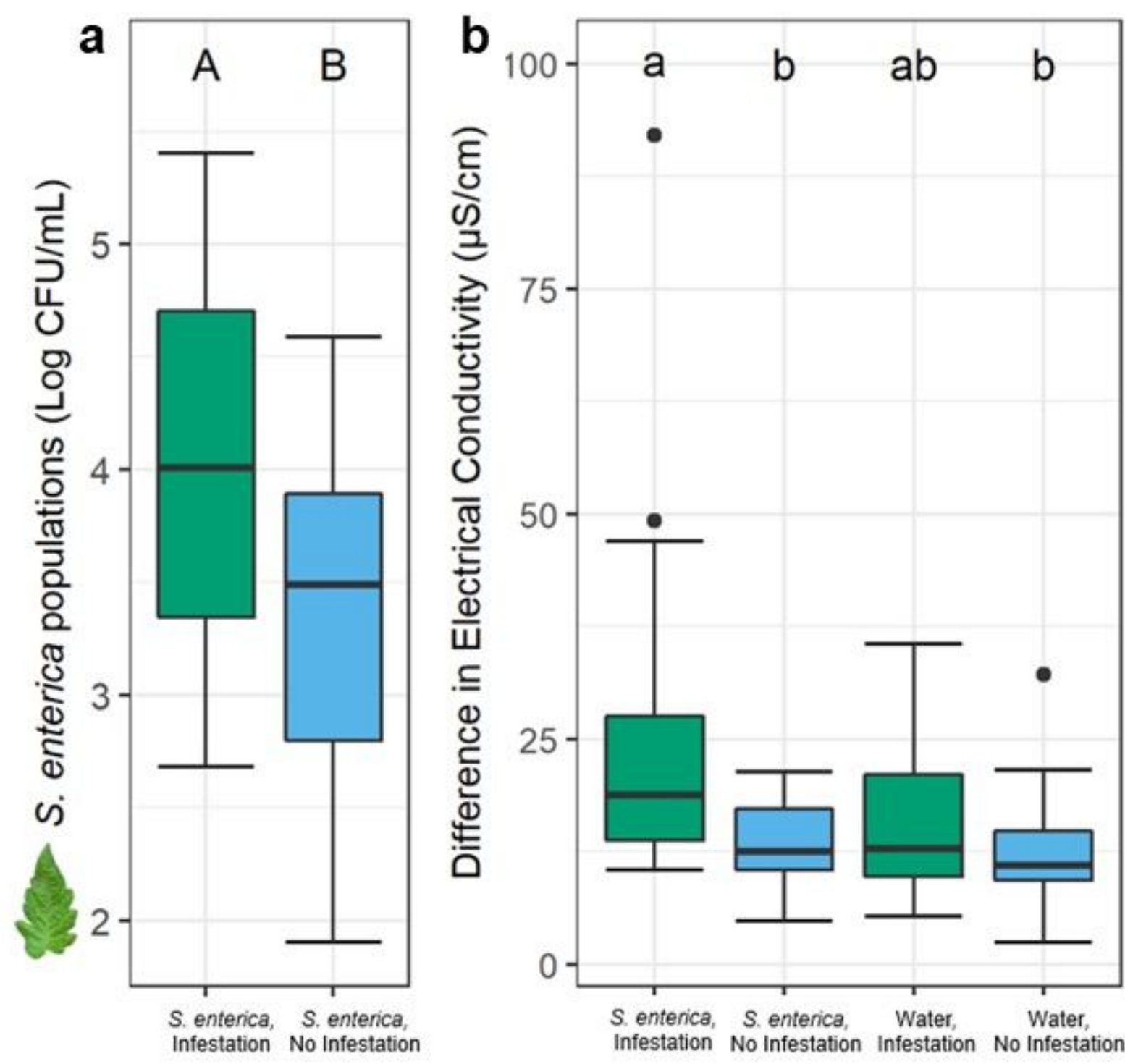

Status of Insect Infestation

Absent

Present

\section{Figure 3}

Macrosteles quadrilineatus infestation on S. enterica inoculated tomato plants led to heightened bacterial populations and electrical conductivity than plants absent insect infestation. Plants inoculated with either S. enterica or water (mock) were infested by adult M. quadrilineatus or remained absent of insects. Empty clip cages were applied for treatment groups with no infestation. Electrical conductance was calculated by subtracting the final from the initial measurement for damaged and undamaged leaf discs and were used to evaluate the extent of electrolyte leakage over six hours. Each treatment group contains combined data from the tip, middle, and basal regions of leaves. Salmonella enterica populations were measured on water inoculated leaves but yielded $0 \mathrm{CFU}$ and were thus excluded from the figure. Letters above boxplots indicate significant differences between treatment groups within each experiment $(\mathrm{P}<$ $0.05)$ as detected by a one-way ANOVA. Singular dots represent an outlier point. 

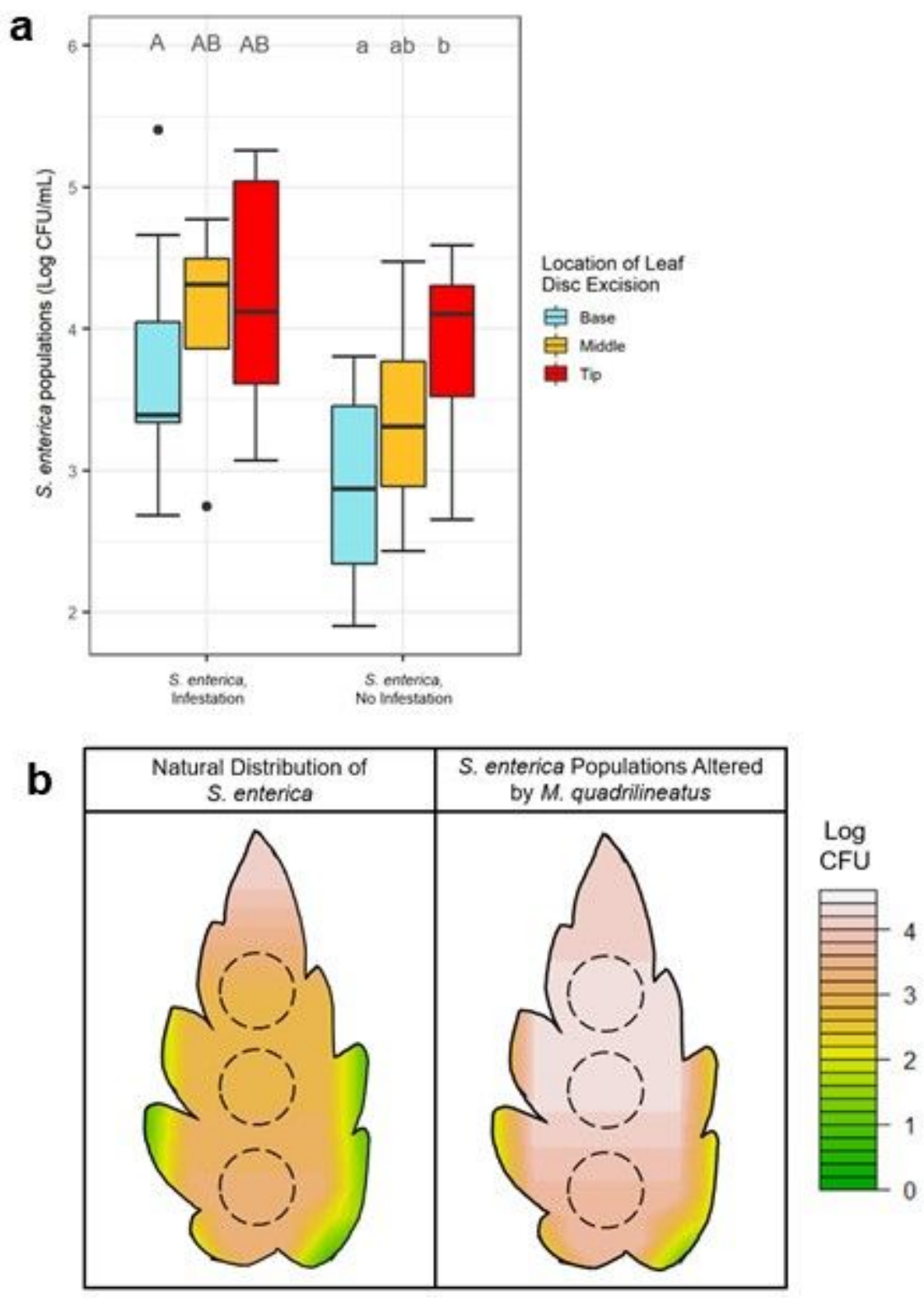

\section{Figure 4}

Adult M. quadrilineatus infestation on S. enterica inoculated tomato plants resulted in a redistribution of bacterial populations. In the absence of insects, $\mathrm{S}$. enterica populations are significantly higher at the tips of leaflets $(P<0.05)$; however, after insect infestation, $S$. enterica populations are approximately uniform across the tip and middle (Fig. $4 a-b$ ) regions. The $2 \times 2$ factorial experiment included S. enterica or water inoculated plants that were either infested, or not infested by M. quadrilineatus. Empty clip cages were applied for treatment groups with no infestation. Leaf discs were excised from the tip, middle and basal regions of leaflets. Salmonella enterica populations were also measured on water inoculated leaves but yielded $0 \mathrm{CFU}$, and were thus excluded from the figure. An interpolation graph was created to depict the shift in bacterial populations (Log CFU) over a 72 hour post-infestation period. Singular dots represent an outlier point. Letters above boxplots indicate significant differences between leaf treatment groups within an insect infestation treatment $(P<0.05)$, as detected by a one-way ANOVA. 




Figure 5

The proportion of M. quadrilineatus that emigrate away from leaflets inoculated with S. enterica increase over 48 hours. Tomato leaflets were inoculated with S. enterica exclusively at the basal (a), middle (b) or tip (c) regions or were entirely submerged during inoculation (Whole; e). Similarly, one group of tomato leaflets were entirely inoculated by water (Water; d). One hour after S. enterica inoculation, five leafhoppers were placed in a container encasing one tomato leaflet, still attached to the plant. Observations were taken 2, 24, and 48 hours after the initial infestation period. The proportion of insects within each treatment represent means from across three experimental replicates between four plants ( $\mathrm{N}$ $=60$ adult $\mathrm{M}$. quadrilineatus). 


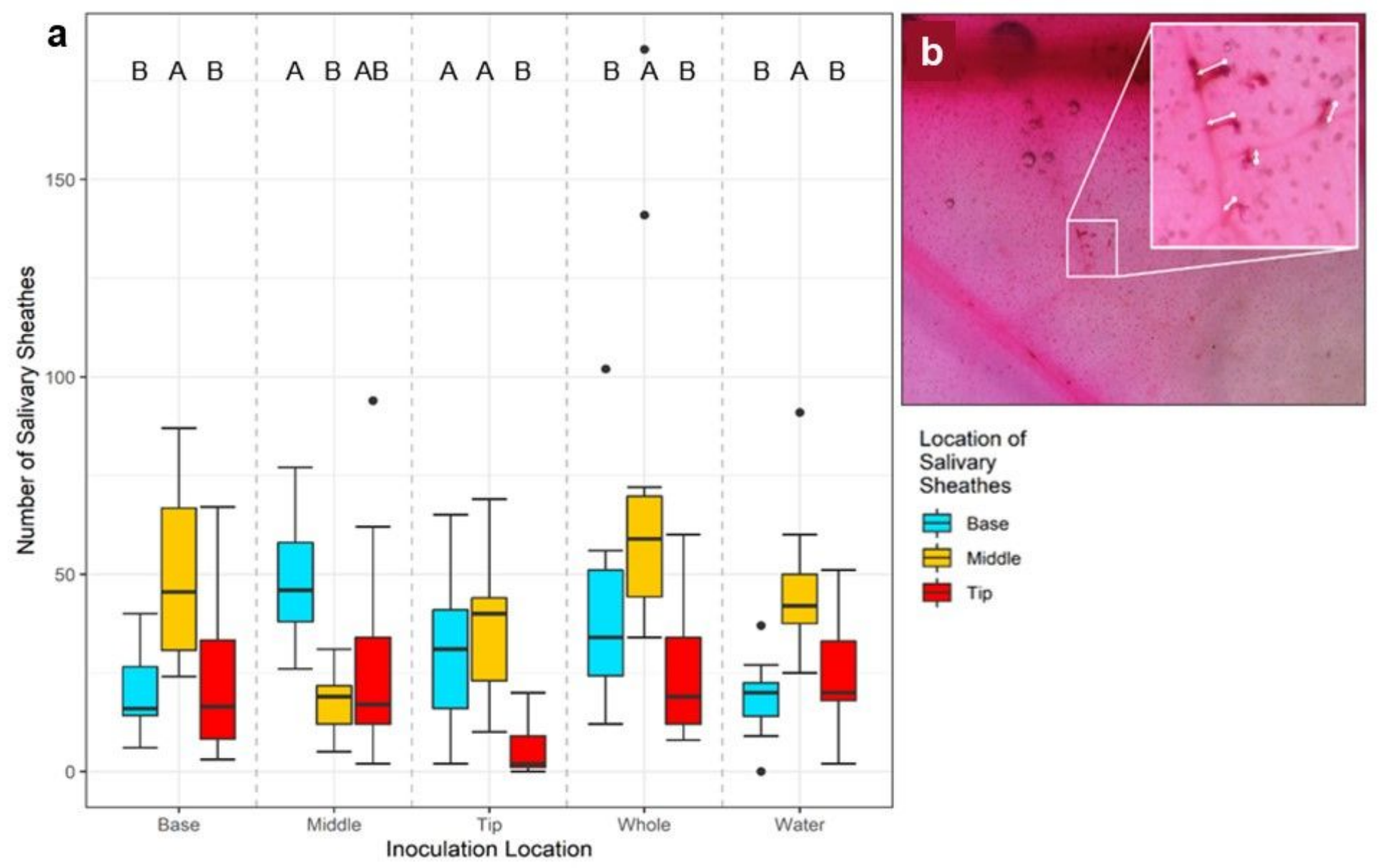

\section{Figure 6}

Salivary sheath distribution across tomato leaflets in response to S. enterica inoculations applied to different regions of plants, excluding those found on the primary and secondary veins. Tomato leaflets were inoculated with S. enterica exclusively at the basal, middle or tip regions, or were entirely submerged during inoculation (Whole). Similarly, one group of tomato leaflets were entirely inoculated by water (Water). One hour after S. enterica inoculation, five adult M. quadrilineatus were placed in a container encasing one tomato leaflet still attached to the plant. Leaflets were extracted 72 hours post $\mathrm{S}$. enterica inoculation and were subjected to staining and clearing procedures to count total salivary sheaths (a). Post clearing and staining procedures, salivary sheathes appeared as dark red in contrast to the pink leaflets (b). Salivary sheathes from three experimental replicates ( $n=60$ leaflets) are combined and represented above. Letters above boxplots indicate significant differences between leaf treatment groups within an insect infestation treatment $(P<0.05)$. 

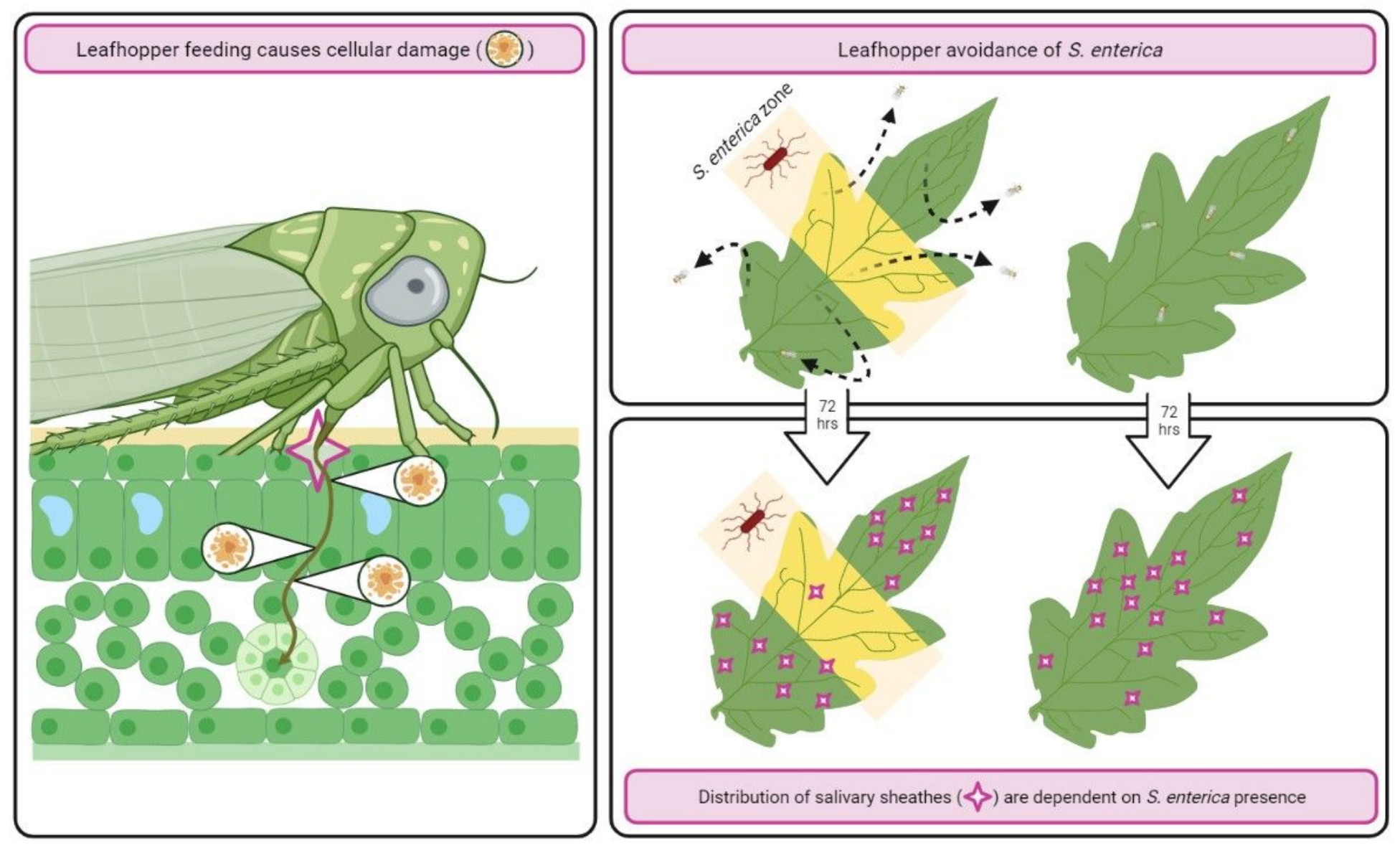

Figure 7

The presence of S. enterica alters $M$. quadrilineatus feeding behaviors. Infestation by M. quadrilineatus on S. enterica inoculated tomato plants resulted in significantly greater rates of localized cellular damage and bacterial populations than uninfested leaflets (left panel). Over a 48-hour period of infestation, cohorts of M. quadrilineatus migrated away from tomato leaflets with partial, or entire, S. enterica inoculation (top). Cleaning and staining procedures 72-hours afterwards demonstrated that zones of bacterial inoculation contained the least amount of salivary sheathes indicating an aversion to $\mathrm{S}$. enterica within the phyllosphere (bottom). Image created through Biorender (biorender.com).

\section{Supplementary Files}

This is a list of supplementary files associated with this preprint. Click to download.

- SupplementalFiguressubmitted8.24.pdf 